\title{
OPEN Temperature and interlayer coupling induced thermal transport across graphene/2D-SiC van der Waals heterostructure
}

\author{
Md. Sherajul Islam ${ }^{1,3 凶}$, Imon Mia ${ }^{1}$, A. S. M. Jannatul Islam ${ }^{1}$, Catherine Stampfl ${ }^{2}$ \& \\ Jeongwon Park ${ }^{3,4}$
}

Graphene based two-dimensional (2D) van der Waals (vdW) materials have attracted enormous attention because of their extraordinary physical properties. In this study, we explore the temperature and interlayer coupling induced thermal transport across the graphene/2D-SiC vdW interface using non-equilibrium molecular dynamics and transient pump probe methods. We find that the in-plane thermal conductivity $\kappa$ deviates slightly from the $1 / T$ law at high temperatures. A tunable $\kappa$ is found with the variation of the interlayer coupling strength $\chi$. The interlayer thermal resistance $R$ across graphene/2D-SiC interface reaches $2.71 \times 10^{-7} \mathrm{Km}^{2} / \mathrm{W}$ at room temperature and $\chi=1$, and it reduces steadily with the elevation of system temperature and $\chi$, demonstrating around $41 \%$ and $56 \%$ reduction with increasing temperature to $700 \mathrm{~K}$ and a $\chi$ of 25 , respectively. We also elucidate the heat transport mechanism by estimating the in-plane and out-of-plane phonon modes. Higher phonon propagation possibility and Umklapp scattering across the interface at high temperatures and increased $\chi$ lead to the significant reduction of $R$. This work unveils the mechanism of heat transfer and interface thermal conductance engineering across the graphene/2D-SiC vdW heterostructure.

The performance of nano-electronics, optoelectronics, and phononic devices relies strongly on the efficiencies of heat energy dissipation ${ }^{1-3}$. The effectiveness of heat energy dissipation is firmly based on phonon transportation ability because phonons are the key thermal energy carriers in semiconducting and insulating materials $\mathrm{s}^{4,5}$. Overpopulated phonons can be caused by the high electrical flow of current, which in effect dramatically enhances electron-phonon scattering as well as phonon-phonon scattering. These processes play into the joule heating effects of regional heat localization and develops local thermal hot spots ${ }^{6,7}$. Failure to effectively dissipate such a robust amount of generated heat will result in reduced performance, types of defects, and even material disruption. For effective development and design of nano-electronic and optoelectronic devices, it is therefore very important to know the device heat transport characteristics. Of late, two-dimensional (2D) materials have acquired enormous interest with regard to solving the problem of heat-energy dissipation and providing an efficient approach to handle the thermal transport phenomenon in nanoscale devices ${ }^{8-17}$. $2 \mathrm{D}$ materials offer appealing electronic, mechanical, and phononic properties, which are highly demanded for nano-electronics, optoelectronics, and phononic devices ${ }^{18-23}$.

On the other hand, advanced structures of 2D materials where two or more atomic sheets are held together by a van der Waals forces, and thus form hybrid structures known as van der Waals heterostructures (vdWHs), can retain their all stacked monolayer properties ${ }^{24-33}$. In this regard, numerous vdWHs have been proposed using graphene and related 2D materials with superior electronic properties, exceptional thermal characteristics, unique mechanical properties as well as other physical properties. In particular, the graphene/2D-SiC vdWH has recently been explored with exotic thermal behavior and unique electronic properties being reported ${ }^{2,28}$. In our earlier study, we also reported that graphene/2D-SiC ${ }^{2}$ shows exceptional thermal transport across the interface to mitigate the heat dissipation problem in next generation nanoelectronic devices. However, thermal transport at the nanoscale shows diverse temperature dependent behaviors ${ }^{34-36}$. At high temperatures, the thermal twitching

${ }^{1}$ Department of Electrical and Electronic Engineering, Khulna University of Engineering andTechnology, Khulna 9203, Bangladesh. ${ }^{2}$ School of Physics, The University of Sydney, Sydney, NSW 2006, Australia. ${ }^{3}$ Department of Electrical and Biomedical Engineering, University of Nevada, Reno, NV 89557, USA. ${ }^{4}$ School of Electrical Engineering and Computer Science, University of Ottawa, Ottawa, ON K1N 6N5, Canada. ${ }^{\square}$ email: sheraj_kuet@ eee.kuet.ac.bd 
characteristics increase considerably and therefore phonon anharmonicity increases significantly, leading to a decrease in thermal conductivity $\kappa$. Usually, the $\kappa$ obeys the $1 / T$ rule in single atomic crystals such as graphene, phosphorene, and stanene at high temperatures ${ }^{11,37-39}$. However, some binary atomic structures exhibit a peculiar, slowly diminishing characteristic of $\kappa$ with increasing temperature owing to the overwhelming impact of highfrequency optical phonons $s^{35,36}$. It is thus crucial to know what types of temperature dependent behavior could be found from this promising graphene/2D-SiC vdWH. In addition, the compact structure of thermal interface materials in vdWHs may change the contact pressure variations, which can significantly hinder the thermal transport performance ${ }^{40-43}$. Along with the temperature effects, a detailed insight into contact pressure effects is also required to efficiently lower the thermal resistance across graphene/2D-SiC interface.

Here, we explore the temperature and interface contact pressure effects on thermal transport across the graphene/2D-SiC interface employing reverse non-equilibrium molecular dynamics (RNEMD) simulations and the transient pump-probe (TPP) method. The thermal resistance across the graphene/2D-SiC interface and inplane thermal conductivity have been evaluated by varying the temperature from 100 to $700 \mathrm{~K}$ and the coupling strength from 0.1 to 25 . When estimating thermal conductivity at lower temperatures, classical MD simulations neglect quantum effects. The thermal transport at the interface incorporating the quantum correction effect is also investigated. Furthermore, the key mechanisms of heat transfer in the in-plane and out-of-plane directions of the heterobilayer are explored estimating various phonon modes.

\section{Computational details}

The graphene/2D-SiC heterostructure was built using a supercell consisting of a lateral periodicity of $2 \times 2$ for graphene and $3 \times 3$ for $2 \mathrm{D}-\mathrm{SiC}$, respectively. To maintain commensurability, the graphene/2D-SiC lattice parameter was set to $a_{\mathrm{C} / \mathrm{SiC}}=3 a_{\mathrm{SiC}}=9.285 \AA$, imposing a $1 \%$ tensile strain on graphene. To attain a large structural system, the supercell structure was repeated in the $\mathrm{x}$ and $\mathrm{y}$ axes. The interlayer interaction between two layers was described using the Lennard Jones (LJ) potential according to

$$
V(r)=4 \chi \varepsilon\left[\left(\frac{\sigma}{r}\right)^{12}-\left(\frac{\sigma}{r}\right)^{6}\right] ; r<r_{c},
$$

where $r, \varepsilon, r_{c}$, and $\sigma$ denote the interatomic distance, energy, cut off distance and length parameters, respectively. The interface interaction parameter is indicated by $\chi$, and it may be changed to control the interface strength. The parameters $\varepsilon_{C-C}=4.560 \mathrm{meV}, \varepsilon_{S i-C}=8.909 \mathrm{meV}, \sigma_{C-C}=3.431 \AA$ and $\sigma_{S i-C}=4.067 \AA$ were chosen from Ref ${ }^{44}$. The interlayer coupling $\chi$ and cutoff distance were set at 1 and $10 \AA$, respectively. Initially, the interlayer separation between graphene and 2D-SiC layer was selected to be $3.4 \AA$. The spacing, however, is automatically changed after the structure is relaxed. All the directions were subjected to periodic boundary conditions (i.e., $x, y, z)$ to eradicate the edge effect. A $10 \mathrm{~nm}$ thick vacuum distance in the $z$-direction was also used to remove atomic contact with the system image. The dimension of the structure considered here was $100 \times 10.8 \mathrm{~nm}^{2}$ (length $\times$ width). All the calculations were carried out in the Large Scale Atomic/Molecular Massively Parallel Simulator (LAMMPS) package ${ }^{45}$. Optimized Tersoff potentials formulated by Lindsay et al. ${ }^{46}$ and Erhart et al. ${ }^{47}$ were employed to explain $\mathrm{C}-\mathrm{C}$ and $\mathrm{Si}-\mathrm{C}$ interactions in the graphene and $\mathrm{SiC}$ layers, respectively. These potentials were previously utilized to accurately measure the thermal transport behavior of graphene and $2 \mathrm{D}-\mathrm{SiC}$.

To quantify the in-plane thermal transport, the RNEMD simulations ${ }^{48}$ were carried out in two phases: the relaxation of the system and the thermal foisting steps. The structure was first brought to an equilibrated position at the desired temperature during the relaxation process. After minimizing the energy with the Conjugated Gradient Algorithm, an isothermal-isobaric (NPT) ensemble simulation was performed for $10^{5}$ time steps to tune the system to the desired pressure and temperature, where each time step $\Delta \mathrm{t}$ was $0.5 \mathrm{fs}$. The velocity-verlet integration method was employed to reach the required temperature. The initial temperature was then raised to the target temperature using 50 ps of microcanonical (NVE) ensemble simulation with a time step of 10 ps. As the temperature was shifted to a new value, the temperature and pressure of the system were further stabilized by applying an additional NPT ensemble simulation for $100 \mathrm{ps}$. The structure was then further subjected to an additional NVE ensemble simulation for 400 ps to check whether the system was relaxed or not and to stabilize the conserved energy. If the temperature of the system does not change with time, indicating that the system is equilibrated at that particular temperature. The quantitative atomic velocity distribution in the system was further estimated and compared to the Maxwell-Boltzmann predictions to confirm that the structure becomes stable. If the structure becomes stable, the velocities of the atoms in the heterostructure follow the Maxwell-Boltzmann distribution as follows:

$$
P_{M}=4 \pi v^{2}\left(\frac{m}{2 \pi K_{B} T}\right)^{\frac{3}{2}} e^{-\frac{m v^{2}}{2 K_{B} T}}
$$

here, $P_{M}, v, K_{B}$, and $m$ represent the probability, velocity, Boltzmann constant, and mass of the atom. We also monitor the process temperature over time to ensure that the temperature stays stable. After the system has been relaxed, the NEMD simulation was done to determine the in-plane thermal conductivity of the graphene/2D-SiC vdWH using Fourier's law as follows:

$$
k_{x}=-\frac{J_{x}}{\nabla T}
$$

where $\nabla T$ is the temperature gradient, and $J_{x}$ signifies the $x$-direction heat flux. A hot and cold chunk with a width of $20 \AA$ was introduced in the system positioning at $L / 4$ and $3 L / 4$ locations, respectively, where $L$ represents 


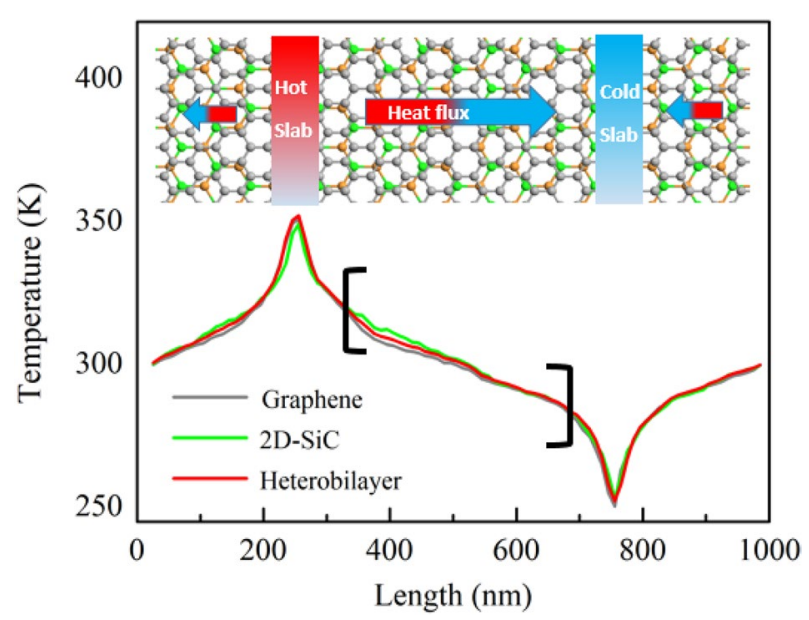

Figure 1. Atomic configuration and NEMD application procedure for the graphene/2D-SiC hetero-bilayer. Periodic boundary conditions are applied in the lateral $x$ and $y$ directions. Free boundary condition is used in the $z$ direction. Temperature distributions of graphene, 2D-SiC and bilayer at steady state.

the length. The heat flux passing through the system can be computed as $J_{x}=\frac{\Delta \varepsilon}{2 A M t}$, where $\Delta \varepsilon$ is the energy difference of the thermostat at every $M$ (heat exchange frequency) time steps, and $A=w h$, is the cross-sectional area. The value of $\mathrm{M}$ was set to $1000(500 \mathrm{fs})^{7,34}$. $t$ denotes the time step of the MD simulations. Following the imposition of heat energy, the time-averaged temperature distribution was calculated to perform NVE ensemble simulation for an additional $24 \times 10^{6}$ time steps (i.e., $1200 \mathrm{ps}$ ) from which $\Delta T$ was computed.

Finally, the thermal transport across interface was investigated by calculating the interfacial thermal resistance $R$. We employed TPP to probe the $R$ at the heterobilayer interface ${ }^{49}$. The schematic of the TPP technique is portrayed in Fig. S1 (supplementary information). The interface heat transport for several vdWHs has been evaluated by this technique in past studies ${ }^{40-43}$. The system was relaxed at a specific temperature to calculate the $R$ across the graphene/2D-SiC interface using the same steps as the $\kappa$ measurement portion. Following the system's relaxation, the graphene layer was subjected to a $50 \mathrm{fs}$ ultrafast heat pulse. As a result, the graphene layer's temperature rises, while the $2 \mathrm{D}$-SiC layer's temperature stays unchanged. Once the heat impulse is withdrawn, the graphene temperature will gradually drop, while the temperature of the $\mathrm{SiC}$ sheet would gradually rise. The temperature evolution of graphene $\left(T_{\mathrm{G}}\right)$ and $2 \mathrm{D}-\mathrm{SiC}\left(T_{2 \mathrm{D}-\mathrm{SiC}}\right)$ was tracked throughout the entire thermal equilibrium procedure. At each step, the total energy of graphene $\left(E_{t}\right)$ was also collected in order to calculate the $R$ according to ${ }^{2}$

$$
\frac{\partial E_{t}}{\partial t}=\frac{A_{R} \cdot\left(T_{G}-T_{2 D-S i C}\right)}{R}
$$

where $A_{\mathrm{R}}$ is the graphene/2D-SiC contact area. To reduce data noise, the data points acquired at each time step were averaged after every 100 time steps.

\section{Results and discussion}

Observing the time dependent system temperature and the atomic velocities, the stability of the developed vdWHs was confirmed at steady-state conditions. As revealed in Fig. S2a (supplementary information), the temperature does not change with time during the relaxation state, especially in the NVE ensemble state, indicating that the structure is in thermal equilibrium. Figure S2b (supplementary information) shows a comparison of the graphene and 2D-SiC atomic velocity distributions with the Maxwell theoretical prediction. The MD simulation results agree with the Maxwell distribution very precisely, which further justifies that the structure is in thermal equilibrium or steady-state condition. The projected temperature distribution profile at steady-state along the $\mathrm{x}$-direction of the heterobilayer is presented in Fig. 1. Here, $T+\Delta T$ and $T-\Delta T$ are used to control the temperature of the hot and cold chunks, respectively, where $\Delta T$ is around $50 \mathrm{~K}$. A rapid temperature fall can be seen near the heating and cooling areas. Similar results have been obtained in NEMD simulations for a variety of systems ${ }^{6,34}$. There are rapid energy transfers between potential and kinetic energies near the hot and cold points. $\nabla T$ is calculated from the average temperature of the regions indicated by the black brackets as displayed in Fig. 1 . In contrast to heterobilayer, graphene and $2 \mathrm{D}$-SiC temperature distributions are also shown, demonstrating the distribution of heterostructure stays within the graphene and $2 \mathrm{D}$-SiC layers. Graphene, $2 \mathrm{D}-\mathrm{SiC}$, and the heterobilayer $\nabla T$ 's are calculated to be $0.336,0.426$, and $0.378 \mathrm{~K} / \mathrm{nm}$, respectively.

To investigate the temperature effect on $\kappa$, a widely varying temperature, ranging from $100 \mathrm{~K}$ to $700 \mathrm{~K}$ and $\chi=1$, is considered. Figure 2 demonstrates the temperature dependence of in-plane $\kappa$ for the vdWH as well as the $2 \mathrm{D}$ sheet of graphene and $\mathrm{SiC}$. The in-plane thermal transport behavior of vdWHs is largely affected by the system thickness. Although, the height of a 2D system is normally taken as the thickness of that specific monolayer ${ }^{26,50,51}$, the total thickness of a vdWH system should be estimated carefully since there is an interlayer gap between the two materials. During the investigation, the overall thickness of the vdWH was measured as the summation of 

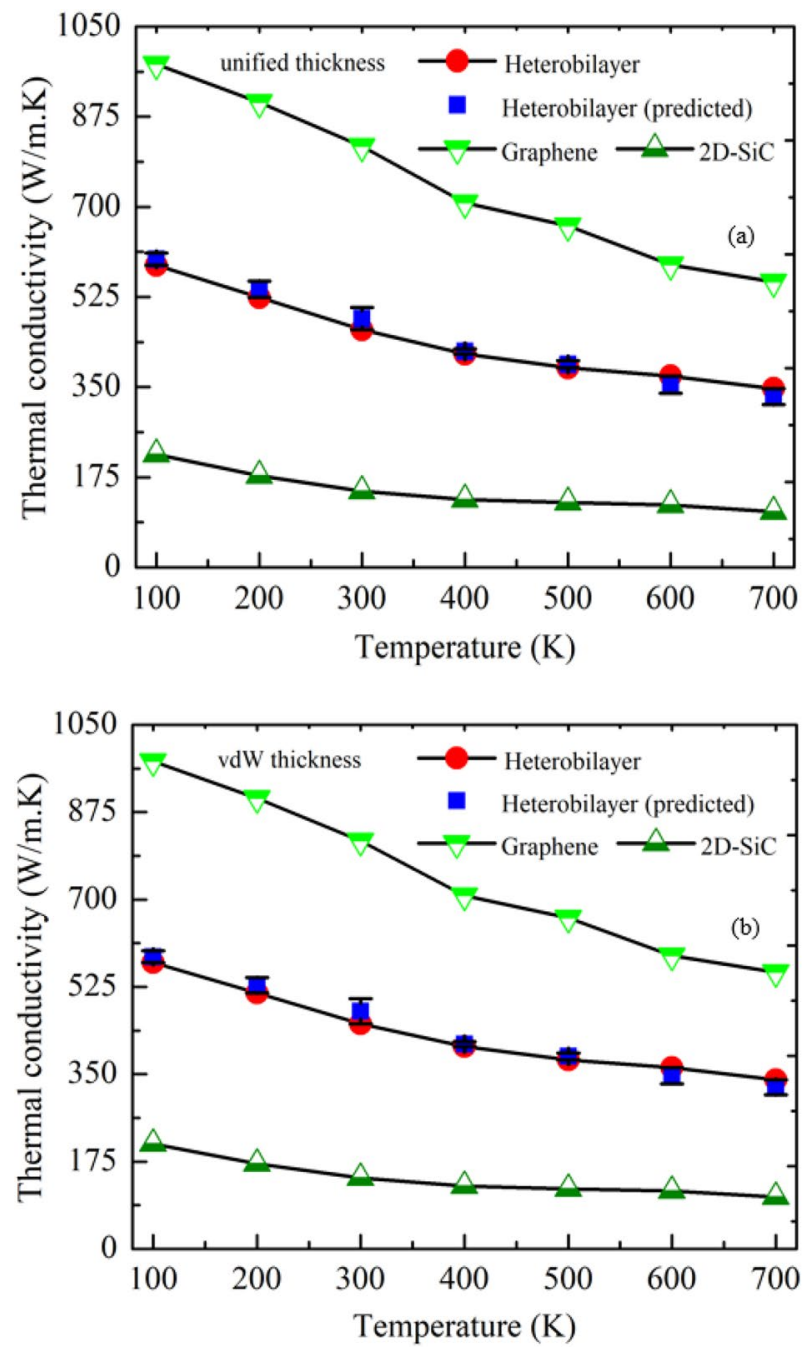

Figure 2. Temperature dependence of thermal conductivity of graphene, $2 \mathrm{D}-\mathrm{SiC}$ and the hetero-bilayer from 100 to $700 \mathrm{~K}$ at (a) unified thickness and (b) vdW thickness (Coupling strength $\chi=1$ ). Each data point is averaged from three independent simulations with different initial conditions.

two distinct material thicknesses. The thicknesses of $2 \mathrm{D}$-SiC and graphene were assumed to be $d_{2 D-S i C}=3.5 \AA$, and $d_{G}=3.35 \AA^{34,52}$. Hence, the total thickness of the graphene/2D-SiC vdWHs was $6.85 \AA$. Nonetheless, Wu et al. ${ }^{53}$ proposed that the sheet thickness of different $2 \mathrm{D}$ configurations does not change with alteration of the material. They discovered that the thickness of the graphene $(3.35 \AA)$ could be applied for computing thermal conductivity in all types of $2 \mathrm{D}$ structures. Consequently, both $d_{G}$ and $d_{2 D-S i C}$ are considered to be $3.35 \AA$. In this perspective, the total thickness of the vdWH is $6.7 \AA$. We counted both of these thicknesses in our study, which we designated as vdW thickness (6.85 ̊) and unified thickness (6.7 ̊).

It is obvious from Fig. 2 that the in-plane $\kappa$ is almost the same for both thicknesses. With increasing temperature, a monotonic decreasing trend of $\kappa$ is observed for both vdWH and individual layer of graphene and $2 \mathrm{D}-\mathrm{SiC}$, as anticipated for phonon mediated heat conduction in nanomaterials. The maximum value of $\kappa$ reaches to $586.47 \mathrm{~W} / \mathrm{mK}$ at $100 \mathrm{~K}$ for the graphene/2D-SiC vdWH, and reduces to $346.81 \mathrm{~W} / \mathrm{mK}$ at $700 \mathrm{~K}$. The maximum drop of $\kappa$ for graphene/2D-SiC vdWH, and individual sheet of graphene and $2 \mathrm{D}-\mathrm{SiC}$ are found as $41.0 \%$, $43.3 \%$, and $39.8 \%$, respectively. Several forgoing studies on 2D materials based vdWH also yielded similar results ${ }^{5,54,55}$. Ahammed et al. ${ }^{5}$ revealed that as the temperature rises from 100 to $700 \mathrm{~K}$, the $\kappa$ of stanene/2D-SiC heterostructure reduces from $\sim 70 \mathrm{~W} / \mathrm{mK}$ to $\sim 24.81 \mathrm{~W} / \mathrm{mK}$. With increasing temperature from 100 to $600 \mathrm{~K}$, the $\kappa$ declines from $\sim 365 \mathrm{~W} / \mathrm{mK}$ to $150 \mathrm{~W} / \mathrm{mK}$ for the stanene/Graphene heterostructure ${ }^{41}, \sim 1964.71 \mathrm{~W} / \mathrm{mK}$ to $\sim 438.19 \mathrm{~W} / \mathrm{mK}$ for the graphene $/ C_{3} N$ heterostructure ${ }^{43}$, and $\sim 9 \mathrm{~W} / \mathrm{mK}$ to $\sim 2 \mathrm{~W} / \mathrm{mK}$ for the stanene/silicene hetero-bilayer ${ }^{56}$, which all are in well agreed with our findings. Moreover, similar outcomes were also reported for heterostructures based on $\mathrm{MoS}_{2}$ and $\mathrm{MoSe}_{2}{ }^{57,58}$.

Though the estimation of $\kappa$ of heterobilayer is based on the Fourier law, we observe that the $\kappa$ digresses from the standard 1/T law at high temperatures. As interatomic force fields have a crucial effect on $\kappa$, we calculate the temperature dependent $\kappa$ behavior for two different potentials. As demonstrated in Fig. 3, $\kappa$ differs slightly from the standard 1/T law for both types of potentials. The amount of divergence from the Fourier law was also calculated, yielding $T^{-0.832}$ and $T^{-0.851}$ for the optimized and initial Tersoff potentials, respectively. This type of 


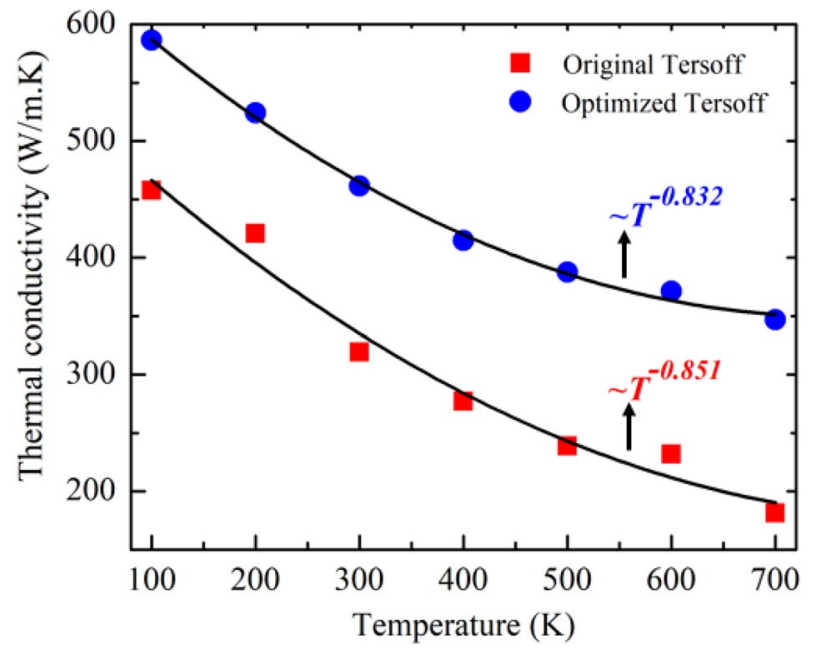

Figure 3. Temperature dependence of the thermal conductivity of the graphene/2D-SiC hetero-bilayer for two different potentials.

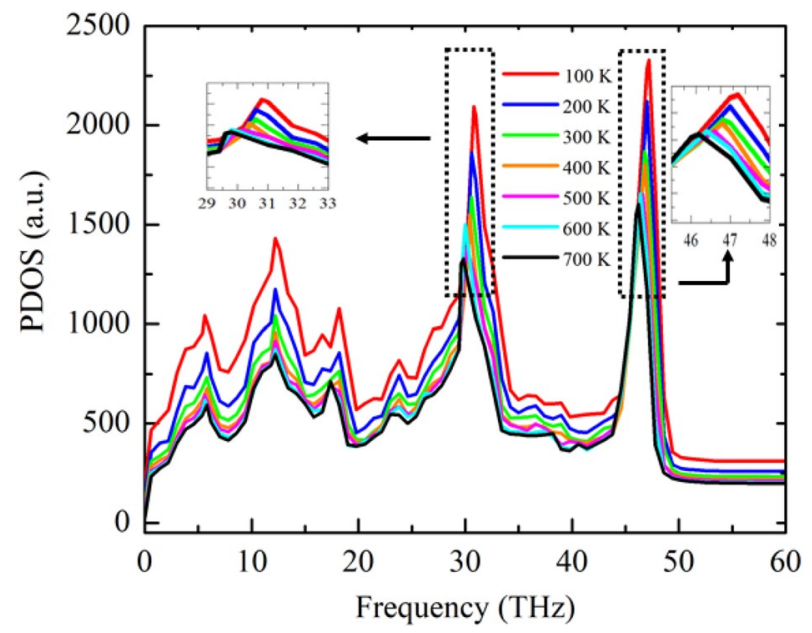

Figure 4. Effect of temperature on the in-plane phonon density of states (PDOS).

slow reduction rate of $\kappa$ at high temperatures has also been observed in $\mathrm{Si} / \mathrm{Ge}$ superlattice ${ }^{59}$, stanene/2D-SiC ${ }^{5}$ vdWH, single layer $2 \mathrm{D}-\mathrm{SiC}$ sheet ${ }^{34}$ and monolayer $\mathrm{GaN}^{35}$. As mentioned in Ref. ${ }^{60}$, the enhanced phonon-phonon Umklapp scattering causes this $1 / \mathrm{T}^{\alpha}$ trend. Moreover, Qin et al. ${ }^{35}$ demonstrated that the contribution of high-frequency phonons may increase with increasing temperature, leading to this slow rate reduction behavior.

To understand this unusual behavior, the temperature dependent phonon spectrum was calculated. The phonon density of states (PDOS) was measured by means of Fourier transform of velocity auto-correlation function $(\mathrm{VACF})^{6}$. We calculated the VACF according to

$$
Z_{\alpha}(t)=\frac{\left\langle V_{i \alpha}(0) \cdot V_{i \alpha}(t)\right\rangle}{\left\langle V_{i \alpha}(0) \cdot V_{i \alpha}(0)\right\rangle}
$$

where $V_{i \alpha}(0)$ and $V_{i \alpha}(t)$ are the $i^{\text {th }}$ particle velocities of element a $(\mathrm{C}$ or $\mathrm{Si})$ at time 0 and $t$, respectively. The PDOS was determined from the VACF according to following formula:

$$
F(\omega)=\frac{1}{\sqrt{2 \pi}} \int_{-\infty}^{\infty} d t e^{i \omega t} Z_{\alpha}(t)
$$

where $F(\omega)$ stands for the phonon spectrum at the vibrational frequency of $\omega$.

Figure 4 depicts the temperature-dependent in-plane PDOS for the graphene/2D-SiC vdWH. The vast portion of in-plane phonons inhabit the high-frequency areas, while a part occupies the low-frequency areas, as shown in Fig. 4, and all of these play a role in the heat energy transmission in this nanomaterial. As the figure demonstrates, the intensity of PDOS peaks reduces with increasing temperature. The reduction of peak intensities 

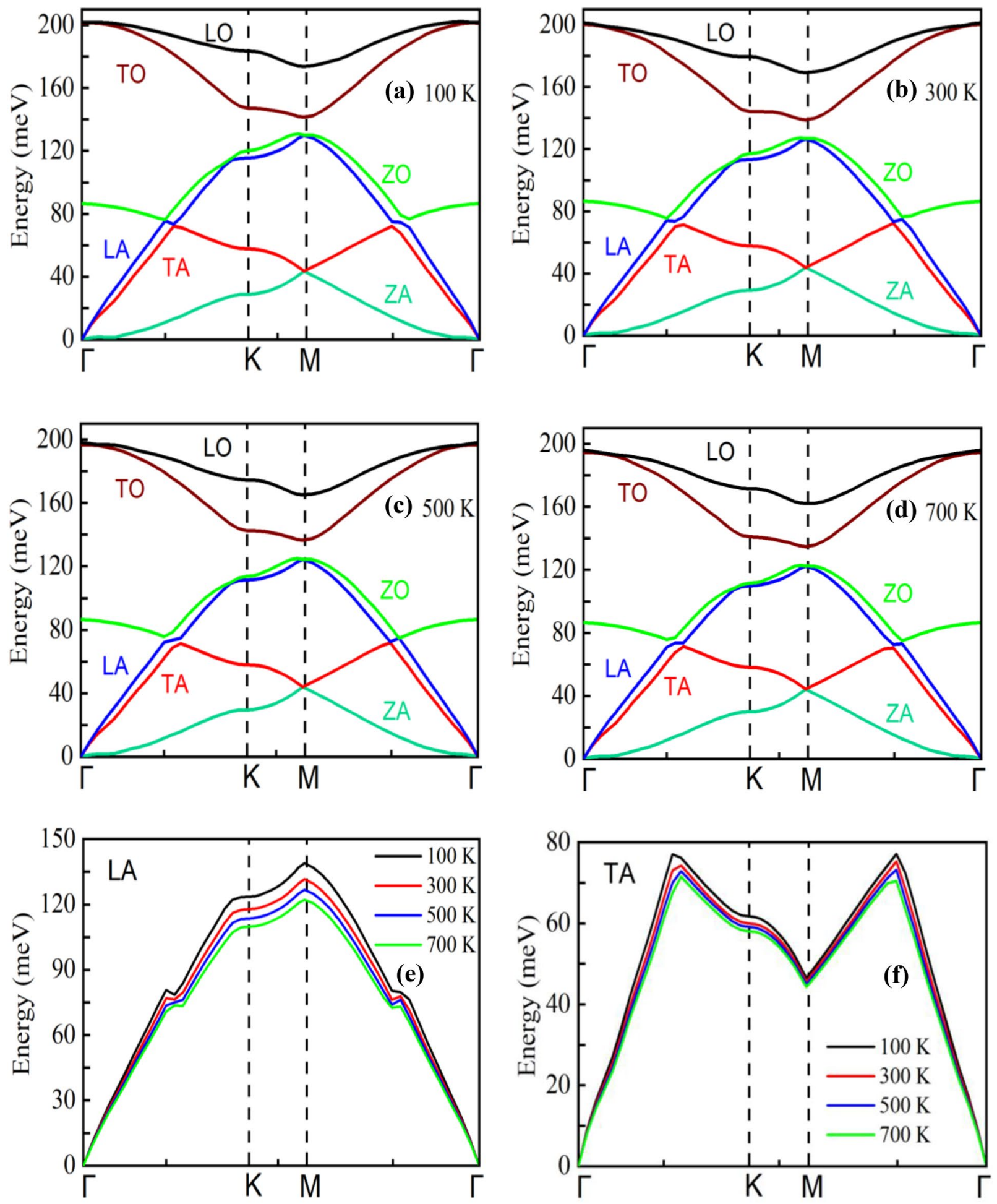

Figure 5. Phonon-dispersion relation for graphene/2D-SiC vdWH at (a) $100 \mathrm{~K}$, (b) $300 \mathrm{~K}$, (c) $500 \mathrm{~K}$, and (d) $700 \mathrm{~K}$. Temperature dependence of (e) LA and (f) TA mode phonons.

complies the decay relation of the $\kappa$ with temperature. Moreover, a redshift is perceived from the fourth peak (29$31 \mathrm{THz})$ and fifth peak (46-48THz) of the PDOS curve. This redshift indicates that the phonon energy decreases at increasing temperatures, which might cause the reduction in $\kappa$ for graphene/2D-SiC vdWH. In $2 \mathrm{D}$ atomic crystals, low-frequency acoustic phonons play a substantial role in the transmission of heat energy. However, at high temperatures, the contribution of low-frequency acoustic phonons are reduced ${ }^{5}$. High-frequency optical phonons predominate at elevated temperatures, resulting in a generation of Umklapp phonon scattering ${ }^{5,34,35}$. 


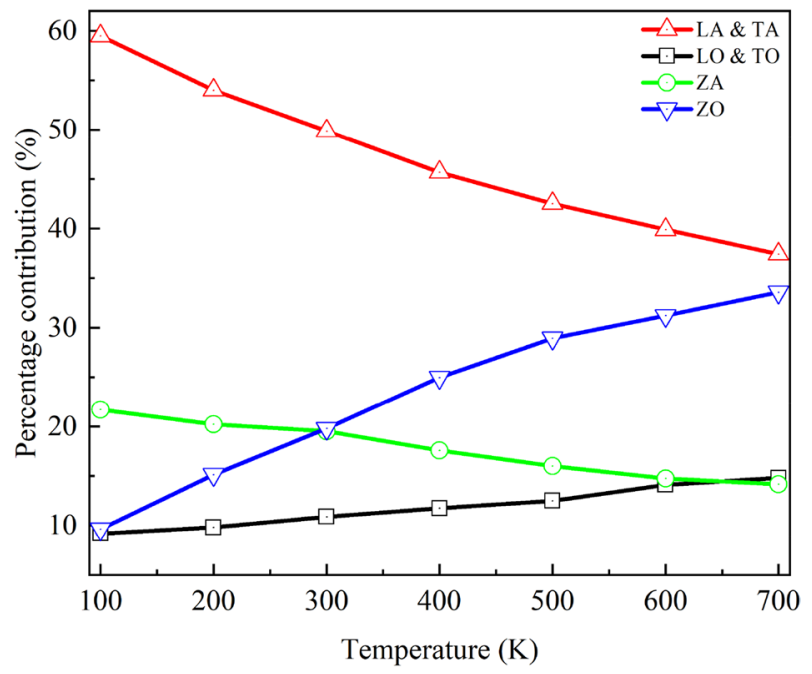

Figure 6. The percentage contribution of various phonon modes as a function of temperature.

Generally, the Umklapp phonon scattering enhances as the temperature of a crystal increases. This leads to the shortening of the mean free path (MFP) of phonons, resulting in a limited phonon heat conduction ${ }^{43}$.

To further explain the temperature effects on $\kappa$, we calculated the temperature dependent phonon-dispersion relation, as depicted in Fig. 5. The phonon-dispersion relation is calculated using the Fix phonon command of LAMMPS package developed by Kong et al. ${ }^{61}$ As shown in Fig. $5 \mathrm{a}-\mathrm{f}$, the low-frequency phonon mode, particularly the LA phonon mode, shows a softening nature with increasing temperature, causing the lower $\kappa$. High-frequency phonon modes such as LO and TO modes also show a downward shifting trend with increasing temperature, which may contribute to a reduction in $\kappa$. We also estimated the percentage contribution of each phonon mode with increasing temperature, as shown in Fig. 6. The contribution of LA and TA modes phonon decreases with increasing temperature. However, the percentage contribution of high frequency mode, especially the ZO phonon, increases significantly. Besides, a slight increase of LO and TO mode phonons is also observed. The enhanced contribution of high-frequency optical phonons at high temperatures may cause this slow rate reduction of $\kappa$. There could be other reasons for this minor deviation, because such a slight deviation is fairly common in defective structures (here heterostructure), as described in Refs ${ }^{62,63}$.

At low temperatures, atomic vibrations remain in a resting place, also known as a frozen state, and remain in that state until the surrounding temperature is raised to a proper level. The sufficient temperature level is known as the Debye temperature level, and the $\kappa$ estimated from the MD simulation up to this point is not fully accurate. Ultimately, to study low-temperature $\kappa$, quantum corrections are required. Using the PDOS of the graphene/2D$\mathrm{SiC}$ heterobilayer, we first calculated the normal mode-specific heat. At constant volume, the specific heat $\left(C_{V}\right)$ can be defined $\mathrm{as}^{34} C_{V}=\frac{3 N \mathrm{~K}_{B} \int_{0}^{\infty} \frac{u^{2} e^{u}}{\left(e^{u}-1\right)^{2}} F(\omega) d \omega}{\int_{0}^{\infty} F(\omega) d \omega}$, where $F(\omega)$ signifies the phonon spectrum, $\mathrm{K}_{B}$ is the Boltzmann constant, and $u=\frac{\hbar \omega}{\mathrm{K}_{\mathrm{B}} T}$. The simulated specific heat for the graphene/2D-SiC vdWH is depicted in Fig. S3 (supplementary information). It is obvious that as the temperature rises, phonon modes are excited, leading to increased specific heat behavior. The $C_{V}$ reaches the steady state due to the complete stimulation of low-frequency phonons at high temperatures. Incorporating the quantum correction at low temperatures $\kappa$ can be estimated according to $\kappa_{q c}=\frac{C_{v}}{3 N K_{B}} \times k_{M D}$, where $k_{M D}$ is the MD simulated thermal conductivity and $\kappa_{q c}$ is the thermal conductivity after quantum correction. The calculated $\kappa_{q c}$ for the optimized and original Tersoff potentials are presented in Fig. 7. For both potentials, the $\kappa_{q c}$ follows a growing trend up to the Debye limit, and then a progressively reducing trend as temperature rises. The calculated Debye limit were found as 257 and $217 \mathrm{~K}$ for the optimized and original Tersoff potentials, respectively. The rise in $\kappa_{q c}$ is owing to the rising nature of the specific heat, coming from the increased quantity of excited phonons up to the Debye limit. Again, up to the Debye limit, the phonon group velocities are assumed to be constant. On the other hand, the saturated nature of $C_{V}$ has a minimal effect on $\kappa_{q c}$ at high temperatures, especially after crossing the Debye threshold. Hence, phonon-phonon scattering, particularly Umklapp scattering at high temperatures, is considered to have a strong $\kappa$-lowering effect. As a result, beyond the Debye limit, the quantum corrected results show a declining trend in thermal conductivity. Furthermore, when compared to the $k_{M D}$, the $\kappa_{q c}$ exposes a relatively limited value up to the Debye limit due to the reduced quantity of excited phonons.

Although we calculated the $\kappa$ considering the linear region of the temperature profile as described in most previous NEMD simulations, a recent study ${ }^{64}$ demonstrated that the nonlinear portion should also not be ignored when calculating thermal transport of finite system. Here, we also calculated the $\kappa$ by considering the average temperature of the thermostats to incorporate the nonlinear region of the temperature profile. The calculated temperature dependent $\kappa$ while considering the average temperature of heat source and sinks is depicted in Fig. S4 (supplementary information). The maximum value of $\kappa$ reaches to $523.93 \mathrm{~W} / \mathrm{mK}, 885.43 \mathrm{~W} / \mathrm{mK}$, and $178.65 \mathrm{~W} /$ 


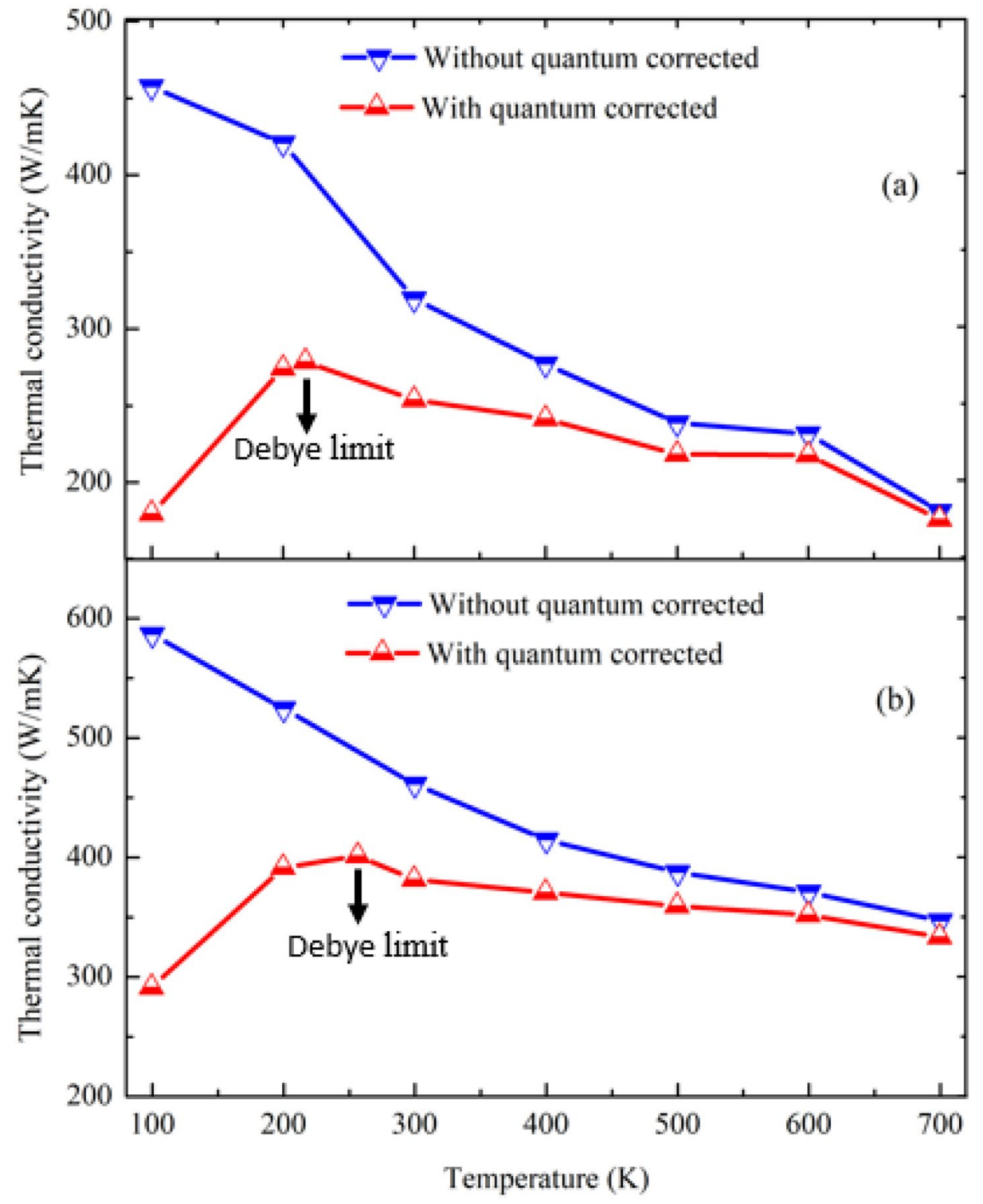

Figure 7. Quantum corrected thermal conductivity of the graphene/2D-SiC hetero-structure [(a) original (b)optimized Tersoff].

$\mathrm{mK}$ at $100 \mathrm{~K}$ for the graphene/2D-SiC vdWH, graphene, and 2D-SiC, respectively, and reduces to $259.10 \mathrm{~W} /$ $\mathrm{mK}, 421.10 \mathrm{~W} / \mathrm{mK}$, and $77.10 \mathrm{~W} / \mathrm{mK}$ at $700 \mathrm{~K}$. It is apparent that at all temperatures, the $\kappa$ is slightly smaller than the estimated $\kappa$ using only the linear portion, which is in line with the demonstration in Ref ${ }^{64}$. This result also implies that the temperature drop close to the thermostat is physical in finite system, as stated in Refs ${ }^{65,66}$.

Improving thermal transport is crucial for novel materials to be used in nanodevices. Various approaches, such as modulating the interface interaction or varying the rotational angle of substrate, have recently been discovered to improve the thermal transport behaviors of vdWHs ${ }^{40-43,49,57,67}$. The interlayer coupling has long been known to have a substantial impact on the thermal transport characteristics. The LJ coupling strength $\chi$ was used to study the interfacial contact pressure dependent $\kappa$ for graphene/2D-SiC vdWH. A wide variety of $\chi$ values ranging from 0.1 to 25 were chosen to determine the coupling strength dependence of $\kappa$. First, the stability of the structures was checked for these diverse values of $\chi$ and then the $\kappa$ dependence of $\chi$ was calculated. Figure 8 represents the variation of the calculated thermal conductivities against $\chi$. Three separate simulations with varied starting conditions were performed and averaged to extract each data point. As $\chi$ increases from 0.1 to 25 , the $\kappa$ of graphene/2D-SiC decreases monotonically from 468.62 to $207.09 \mathrm{~W} / \mathrm{mK}$. The $\kappa$, for the independent layer of graphene, drops dramatically from 842.88 to $357.23 \mathrm{~W} / \mathrm{mK}$, while the $\kappa$ for $2 \mathrm{D}$-SiC drops steadily from 158.32 to $71.82 \mathrm{~W} / \mathrm{mK}$. The flexural mode phonons of $2 \mathrm{D}-\mathrm{SiC}$ constrain the flexural mode phonons of graphene, resulting in a reduction in the $\kappa$ of the graphene/2D-SiC vdWH when compared to the independent layer of graphene or $2 \mathrm{D}-\mathrm{SiC}$ sheet. With increasing $\chi$, restrictions between the flexural mode phonons of the two layers increases as the interaction of the graphene layer and $2 \mathrm{D}-\mathrm{SiC}$ layer is enhanced ${ }^{40-43,67}$. Accordingly, the $\kappa$ of graphene, $2 \mathrm{D}-\mathrm{SiC}$, as well as of the graphene/2D-SiC vdWH decreases as the restriction is increased. 


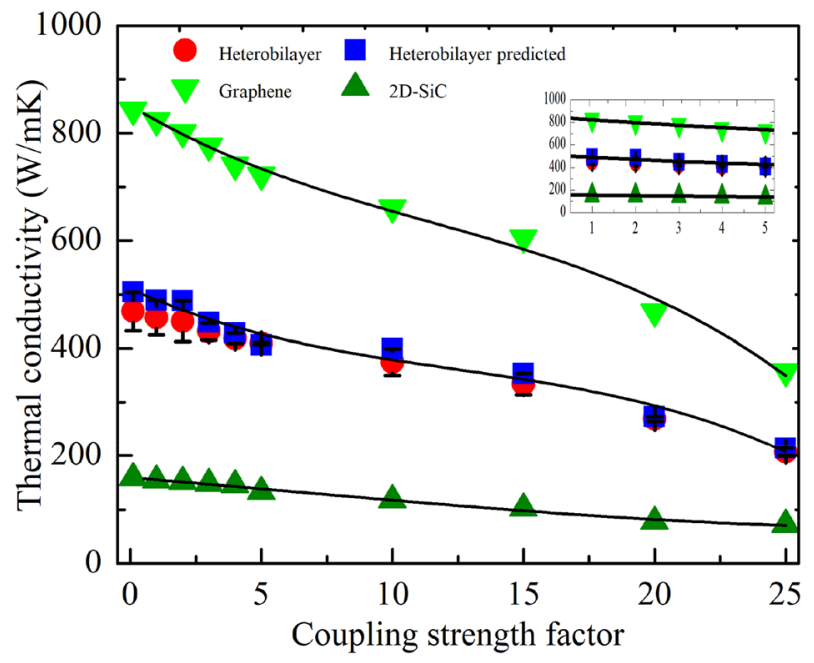

Figure 8. Dependence of in-plane thermal conductivity with interlayer coupling strength $\chi$.
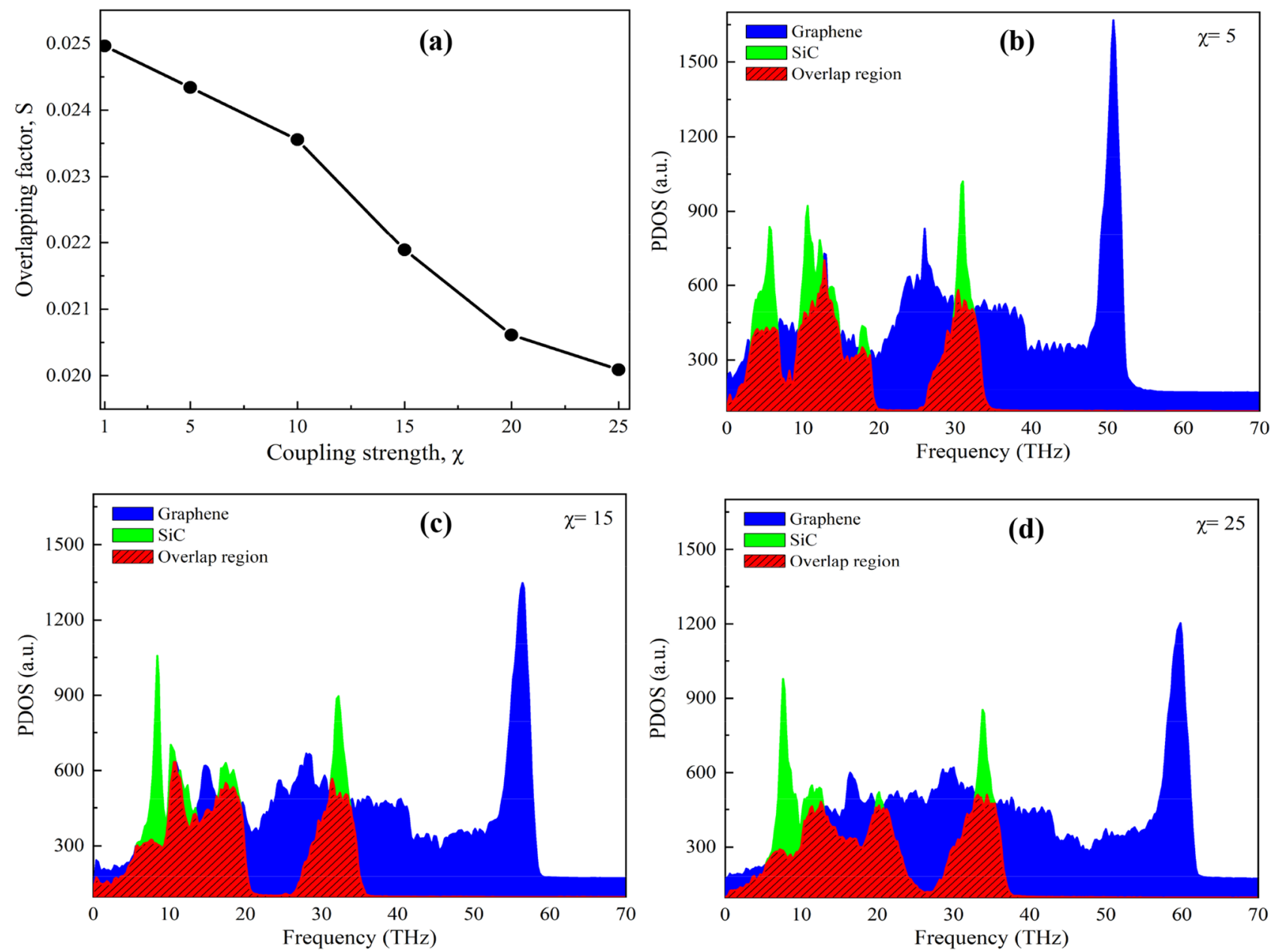

Figure 9. (a) Calculated overlap factor $S$ as a function of interlayer coupling strength $\chi$. (b)-(d) Overlapping region of PDOS between graphene and $2 \mathrm{D}-\mathrm{SiC}$ as a function of $\chi$. The slant areas (red) denote the overlap region between graphene and 2D-SiC PDOS. 


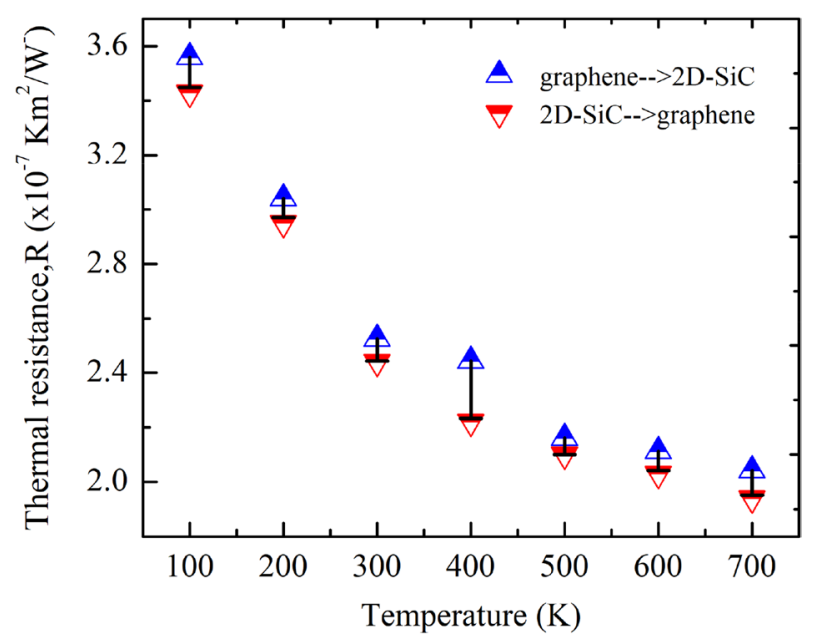

Figure 10. Interfacial thermal resistance variations in the heterobilayer for different temperatures from 100 to $700 \mathrm{~K}$. Here, GRA- $>\mathrm{SiC}$ and $\mathrm{SiC}->$ GRA refer to the direction of heat flow from graphene to $\mathrm{SiC}$ and $\mathrm{SiC}$ to graphene, respectively. There is a slight thermal rectification observed in the out-of-plane thermal conduction for different temperatures.
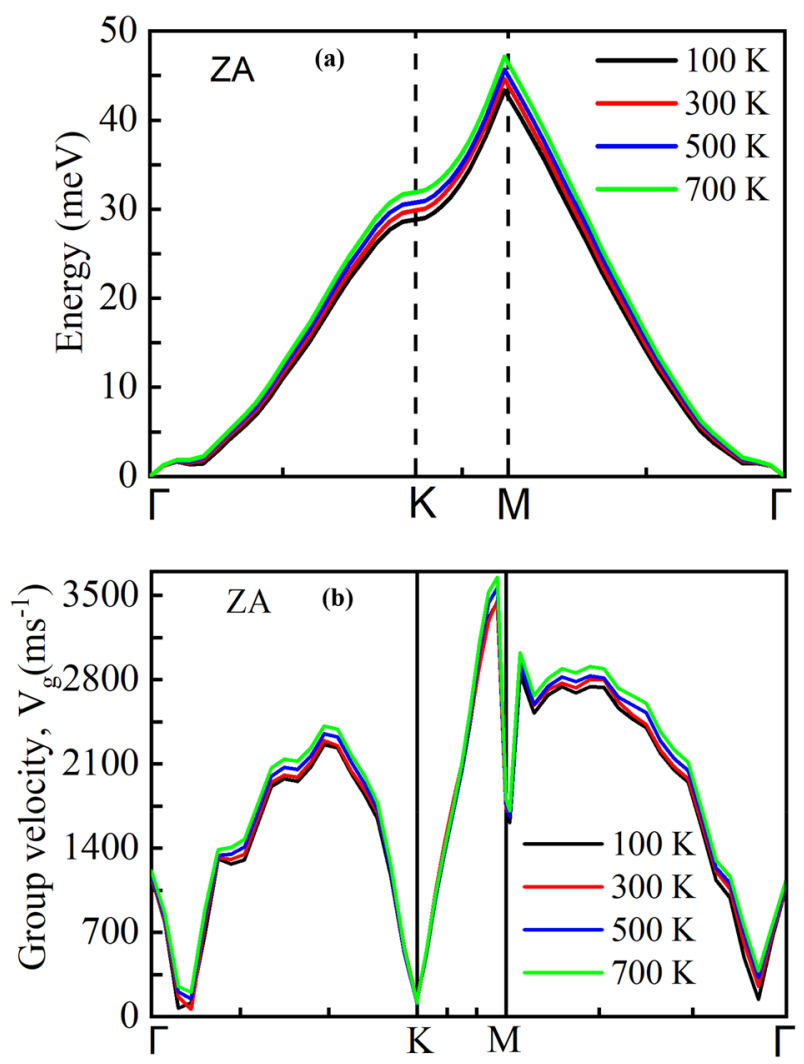

Figure 11. (a) Variation of the phonon-dispersion relation and (b) phonon group velocity of the ZA mode phonon at different temperatures. 


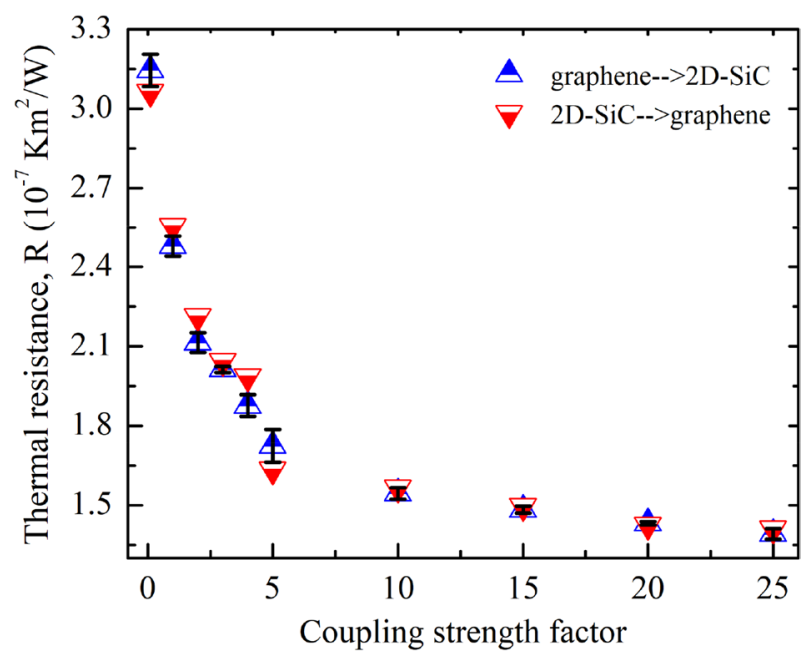

Figure 12. Interfacial thermal resistance variations with different coupling strength factors from 0.1 to 25 . There is no thermal rectification observed in the out-of-plane thermal conduction for different coupling strengths.

Moreover, to have a better understanding of the $\chi$ dependence of $\kappa$, we calculated the phonon overlapping factor $(S)$ of graphene/2D-SiC heterobilayer, which demonstrates the capacity of thermal energy exchanging between graphene and $2 \mathrm{D}-\mathrm{SiC}$ phonon. The following equation is used to compute the overlapping factor $S$ :

$$
S=\frac{\int_{-\infty}^{\infty} F_{\text {graphene }}(\omega) \cdot F_{2 D-S i C}(\omega) d \omega}{\int_{-\infty}^{\infty} F_{\text {graphene }}(\omega) d \omega \cdot \int_{-\infty}^{\infty} F_{2 D-S i C} \cdot d \omega}
$$

where, the subscripts "graphene" and "2D-SiC" denote all the phonon modes of graphene and 2D-SiC, respectively. The $S$ shows a decreasing trend ranging from $\sim 0.026$ to $\sim 0.020$ with the increase of $\chi$ from 0.1 to 25 , as depicted in Fig. 9a-d.The decrease in overlapping area reflects a decrease in the capacity to exchange thermal energy between graphene and $2 \mathrm{D}-\mathrm{SiC}^{68,69}$. Due to the increase of phonon scattering between two layers with increasing $\chi$, the capacity of exchange thermal energy reduces. It can also be observed that the PDOS of graphene is slightly flattened with increasing $\chi$. Such flattening can broaden some phonon modes, potentially reducing phonon lifetime and $\mathrm{MFP}^{70}$. Eventually, this may reduce the overall thermal conductivity of the heterobilayer.

Although 2D materials have superior in-plane heat transport behavior, out-of-plane heat transport is often the barrier for thermal energy exchange due to the poor vdW interaction at the interface. The ability to control the heat flow has led to possible fascinating applications, which strongly depends on the better understanding of the thermal conduction mechanism at the interface. The temperature dependence of $R$ at $\chi=1$ is depicted in Fig. 10. The estimated $R$ reaches to a maximum value $\sim 3.559 \times 10^{-7} \mathrm{Km}^{2} \mathrm{~W}^{-1}$ at $100 \mathrm{~K}$ and monotonically reduces to $\sim 2.041 \times 10^{-7} \mathrm{Km}^{2} \mathrm{~W}^{-1}$ at $700 \mathrm{~K}$. Earlier investigations showed that inelastic scattering affects the most of the energy transport at vdW interface compared to the elastic scattering, at high temperatures ${ }^{57}$. This is because high-frequency phonons may disintegrate into multiple low-frequency phonons at high temperatures, causing the enhancement of inelastic scattering probability. Low-frequency phonons have a better chance of being transmitted through the interface than high-frequency phonons. As a result, phonon transmission coefficients at the interface are enhanced, and $R$ is decreased. Anharmonicity within the materials is discovered to have an essential role in heat transportation at interface by enhancing energy transmission between distinct phonon modes. Wu et al. $^{71}$ explored the influence of anharmonicity in heat transfer across an interface composed of monatomic and diatomic lattices. At higher temperatures, increased anharmonic scattering results in more effective energy redeployment to low-frequency phonons, which can more efficiently transmit heat across the interface, resulting in a reduced $R$. To further explain the temperature effects on $R$, we calculated the temperature dependent out-of-plane phonon-dispersion and phonon-group velocities. As depicted in Fig. 11a, the intensity of ZA mode phonon shows an increasing nature with increasing temperature. It indicates that as the temperature rises, more phonons move from one layer to the next, increasing thermal conductivity in the out-of-plane direction and decreasing $R$. The estimated group velocity for ZA mode phonon as shown in Fig. 11b, also shows an increasing nature with increasing temperature, further verifying the lowering behavior of $R$ as temperature rises.

Next, we study the $\chi$ dependence of $R$ on the graphene/2D-SiC vdWH. Figure 12 illustrates the influence of $\chi$ on $R$ at $300 \mathrm{~K}$. The computed $R$ drops monotonically when $\chi$ varies from 0.1 to 25 . Usually, boosting the $\chi$ increases the interaction at the interface, which enhances the coupling of phonons between $\mathrm{SiC}$ and graphene 

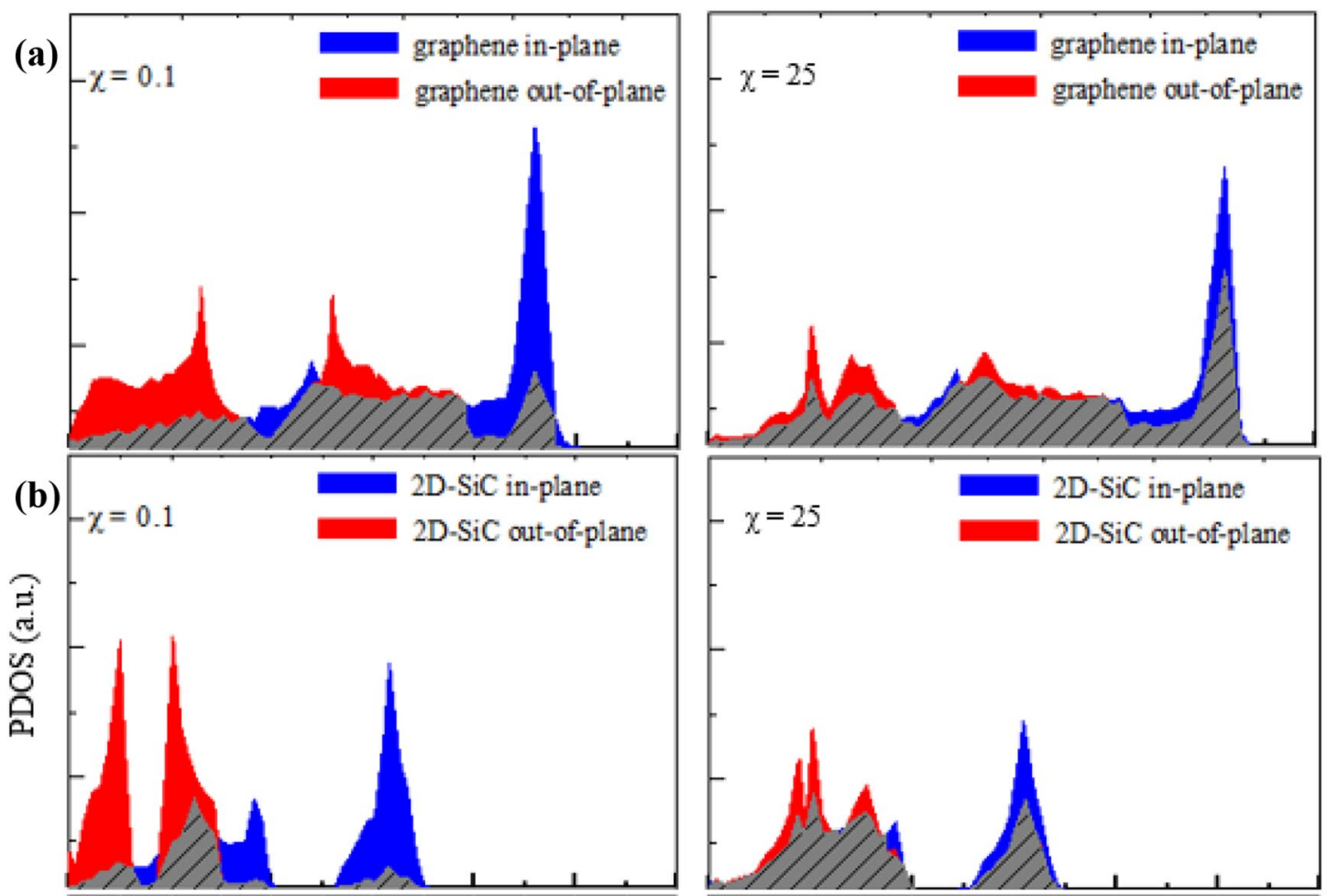

(c)
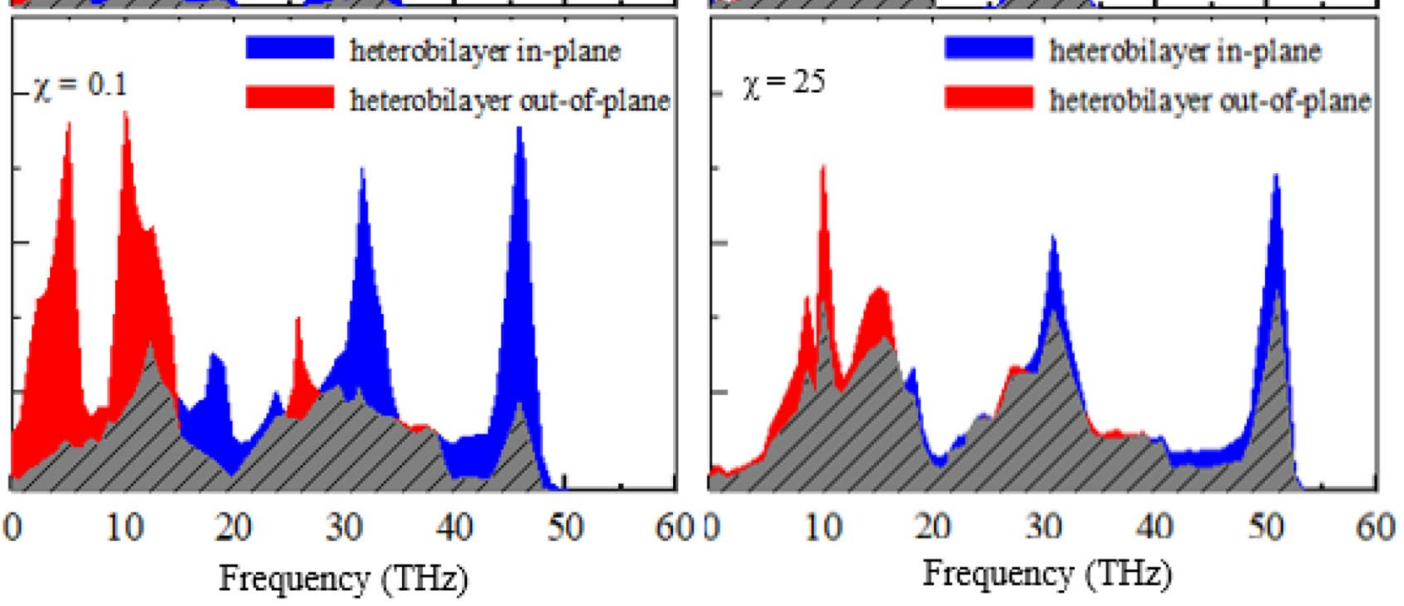

Figure 13. PDOS of (a) graphene, (b) $2 \mathrm{D}-\mathrm{SiC}$, and (c) the graphene/2D-SiC heterobilayer in in-plane and out-of-plane directions with $\chi=0.1$ and $\chi=25$. The slanted line areas denote the overlap of the in-plane and out-of-plane PDOS.

layers and unswervingly raises transmission of phonon probability across the contact. Moreover, atoms in one surface might be scattered more strongly by the other layer's atoms with increasing $\chi$, leading to the increasing of interface scattering. The capability of energy interchange between in-plane and out-of-plane phonons is manifested by calculating the overlap in the PDOS as shown in Fig. 13 . With $\chi=0.1$, the computed $S$ for graphene, $2 \mathrm{D}-\mathrm{SiC}$, and the graphene/2D-SiC vdWH is $0.022,0.026$, and 0.023 , respectively, and increases to 0.026 , 0.034 , and 0.028 when $\chi=25$. It indicates that the interfacial thermal transfer capacity grows when the coupling of in-plane and out-of-plane phonons improves. Because of the scattering of high frequency phonons, the $S$ of graphene has a slight improvement. The $R$ of the system decreases as a result of the improved coupling strength. Therefore, we conclude that interlayer coupling strength may efficiently tune the thermal resistance across the graphene/2D-SiC vdW interface.

\section{Conclusions}

In conclusion, we used NEMD and TPP simulations to systematically investigate temperature and contact pressure induced thermal transport across graphene/2D-SiC vdWH. The computed in-plane $\kappa$ was found to be considerably reduced by system temperature, with maximum reductions of $43.3 \%, 39.8 \%$, and $41.0 \%$ for graphene, $2 \mathrm{D}-\mathrm{SiC}$, and the bilayer, respectively. Different from the typical $1 / T$ law, an abnormal $\kappa$ for the graphene/2D-SiC bilayer was observed at high temperatures. A coupling strength dependent tunable $\kappa$ was observed for this 
heterostructure, which is advantageous for nanodevice production since thermal performance may be adjusted by contact pressure. The calculated $R$ was also found to decrease monotonically with increase of the temperature and coupling strength. The heat conduction mechanism across the heterostructure interface was demonstrated using phonon calculations. The cross-plane phonon interaction between the graphene and $2 \mathrm{D}-\mathrm{SiC}$ layers is revealed to be the primary thermal channel for interfacial heat conduction. The Umklapp scattering converts the high-frequency phonons into more low-frequency phonons, which can subsequently be linked with the phonons in other layer and contributing to interface thermal transport and lowering $R$. Moreover, enhanced phonon coupling with increasing $\chi$ facilitates the heat transfer from graphene to $\mathrm{SiC}$ and thus bring about the decrease in $R$. This study reveals the mechanism of heat dissipation and interfacial engineering for thermal conductance manipulation in the graphene/2D-SiC vdW heterostructure.

\section{Data availability}

The data that support the findings of this study are available from the corresponding author upon reasonable request.

Received: 10 August 2021; Accepted: 24 December 2021

Published online: 14 January 2022

\section{References}

1. Felix, I. M. \& Pereira, L. F. C. Thermal Conductivity of Graphene-hBN Superlattice Ribbons. Sci. Rep. 8, 1-10 (2018).

2. Islam, M. S., Mia, I., Ahammed, S., Stampfl, C. \& Park, J. Exceptional in-plane and interfacial thermal transport in graphene/2D$\mathrm{SiC}$ van der Waals heterostructures. Sci. Rep. 10(1), 1-16 (2020).

3. Xu, X. et al. Length-dependent thermal conductivity in suspended single-layer graphene. Nat. Commun. 5, 1-6 (2014)

4. Balandin, A. A. Thermal properties of graphene and nanostructured carbon materials. Nat. Mater. 10, 569-581 (2011).

5. Ahammed, S., Islam, M. S., Mia, I. \& Park, J. Lateral and flexural thermal transport in stanene/2D-SiC Van Der Waals heterostructure. Nanotechnology 31, 505702 (2020).

6. Islam, A. S. M. J., Islam, M. S., Ferdous, N., Park, J. \& Hashimoto, A. Vacancy-induced thermal transport in two-dimensional silicon carbide: A reverse non-equilibrium molecular dynamics study. Phys. Chem. Chem. Phys. 22, 13592-13602 (2020).

7. Islam, A. S. M. J., Islam, M. S., Islam, M. R., Stampfl, C. \& Park, J. Thermal transport in monolayer zinc-sulfide: effects of length, temperature and vacancy defects. Nanotechnology 32, 435703 (2021).

8. Judek, J. et al. High accuracy determination of the thermal properties of supported 2D materials. Sci. Rep. 5, 1-11 (2015).

9. Zhu, G. et al. Tuning thermal conductivity in molybdenum disulfide by electrochemical intercalation. Nat. Commun. 7, 1-9 (2016).

10. Cui, Y., Qin, Z., Wu, H., Li, M. \& Hu, Y. Flexible thermal interface based on self-assembled boron arsenide for high-performance thermal management. Nat. Commun. 12,1-7 (2021).

11. Peng, B. et al. Low lattice thermal conductivity of stanene. Sci. Rep. 6, 1-10 (2016).

12. Xiao, H. et al. Lattice thermal conductivity of borophene from first principle calculation. Sci. Rep. 7, 1-8 (2017).

13. Luo, Z. et al. Anisotropic in-plane thermal conductivity observed in few-layer black phosphorus. Nat. Commun. 6, 1-8 (2015).

14. Wang, C. et al. Superior thermal conductivity in suspended bilayer hexagonal boron nitride. Sci. Rep. 6, 6-11 (2016).

15. Islam, M. S., Tanaka, S. \& Hashimoto, A. Effect of vacancy defects on phonon properties of hydrogen passivated graphene nanoribbons. Carbon N. Y. 80, 146-154 (2014).

16. Zulfiqar, M., Zhao, Y., Li, G., Li, Z. C. \& Ni, J. Intrinsic Thermal conductivities of monolayer transition metal dichalcogenides MX $2(\mathrm{M}=\mathrm{Mo}, \mathrm{W} ; \mathrm{X}=\mathrm{S}$, Se, Te). Sci. Rep. 9, 1-7 (2019).

17. Peng, B. et al. Phonon transport properties of two-dimensional group-IV materials from ab initio calculations. Phys. Rev. B 94, 245420 (2016).

18. Glavin, N. R. et al. Emerging Applications of Elemental 2D Materials. Adv. Mater. 32, 1-22 (2020).

19. Novoselov, K. S. et al. Electric field in atomically thin carbon films. Science (80-.) 306, 666-669 (2004).

20. Zhao, L., Xu, S., Wang, M. \& Lin, S. Probing the thermodynamic stability and phonon transport in two-dimensional hexagonal aluminum nitride monolayer. J. Phys. Chem. C 120, 27675-27681 (2016).

21. Islam, A. S. M. J., Hasan, M. S., Islam, M. S. \& Park, J. Chirality, temperature, and vacancy effects on mechanical behavior of monolayer zinc-sulfide. Comput. Mater. Sci. 200, 110824 (2021).

22. Islam, A. S. M. J. et al. Anisotropic mechanical behavior of two dimensional silicon carbide: effect of temperature and vacancy defects. Mater. Res. Express 6, 125073 (2019).

23. Islam, A. S. M. J., Akbar, M. S., Islam, M. S. \& Park, J. Temperature- and defect-induced uniaxial tensile mechanical behaviors and the fracture mechanism of two-dimensional silicon germanide. ACS Omega 6, 21861-21871 (2021).

24. Li, M. Y., Chen, C. H., Shi, Y. \& Li, L. J. Heterostructures based on two-dimensional layered materials and their potential applications. Mater. Today 19, 322-335 (2016).

25. Islam, M. S., Mojumder, M. R. H., Ferdous, N. \& Park, J. Germanene/2D-SiC van der Waals heterobilayer: Structural features and tunable electronic properties. Mater. Today Commun. https://doi.org/10.1016/j.mtcomm.2020.101718 (2020).

26. Li, T., Tang, Z., Huang, Z. \& Yu, J. Interfacial thermal resistance of $2 \mathrm{D}$ and $1 \mathrm{D}$ carbon/hexagonal boron nitride van der Waals heterostructures. Carbon N. Y. 105, 566-571 (2016)

27. Wang, X. et al. Electric field effects on the electronic and optical properties in C2N/Sb van der Waals heterostructure. Carbon N. Y. 129, 738-744 (2018).

28. Rashid, A. S. et al. Widely tunable electronic properties in graphene/two-dimensional silicon carbide van der Waals heterostructures. J. Comput. Electron. 18, 836-845 (2019).

29. Ma, H., Babaei, H. \& Tian, Z. The importance of van der Waals interactions to thermal transport in Graphene-C60 heterostructures. Carbon N. Y. 148, 196-203 (2019).

30. Barboza, A. P. M. et al. Graphene/h-BN heterostructures under pressure: From van der Waals to covalent. Carbon N. Y. 155, $108-113(2019)$.

31. Sagar, R. U. R. et al. Large magnetotransport properties in mixed-dimensional van der Waals heterostructures of graphene foam. Carbon N. Y. 159, 648-655 (2020).

32. Sarker, J. D. et al. Tunable electronic properties in bismuthene/2D silicon carbide van der Waals heterobilayer. Jpn. J. Appl. Phys. 59, SCCC03 (2020).

33. Zhao, J., Zeng, H. \& Zhou, X. X $3 \mathrm{~N}(\mathrm{X}=\mathrm{C}$ and $\mathrm{Si})$ monolayers and their van der Waals Heterostructures with graphene and h-BN: Emerging tunable electronic structures by strain engineering. Carbon N. Y. 145, 1-9 (2019).

34. Islam, A. S. M. J. et al. Anomalous temperature dependent thermal conductivity of two-dimensional silicon carbide. Nanotechnology 30, 445707 (2019). 
35. Qin, G., Qin, Z., Wang, H. \& Hu, M. Anomalously temperature-dependent thermal conductivity of monolayer GaN with large deviations from the traditional 1/T law. Phys. Rev. B 95, 195416 (2017).

36. Wang, H., Qin, G., Li, G., Wang, Q. \& Hu, M. Low thermal conductivity of monolayer $\mathrm{ZnO}$ and its anomalous temperature dependence. Phys. Chem. Chem. Phys. 19, 12882-12889 (2017).

37. Cao, A. Molecular dynamics simulation study on heat transport in monolayer graphene sheet with various geometries. J. Appl. Phys. 111, 083528 (2012).

38. Zhang, Y. Y., Cheng, Y., Pei, Q. X., Wang, C. M. \& Xiang, Y. Thermal conductivity of defective graphene. Phys. Lett. Sect. A Gen. At. Solid State Phys. 376, 3668-3672 (2012).

39. Zhang, Y. Y., Pei, Q. X., Jiang, J. W., Wei, N. \& Zhang, Y. W. Thermal conductivities of single- and multi-layer phosphorene: A molecular dynamics study. Nanoscale 8, 483-491 (2016).

40. Hong, Y., Ju, M. G., Zhang, J. \& Zeng, X. C. Phonon thermal transport in a graphene/MoSe2 van der Waals heterobilayer. Phys. Chem. Chem. Phys. 20, 2637-2645 (2018).

41. Hong, Y., Zhu, C., Ju, M., Zhang, J. \& Zeng, X. C. Lateral and flexural phonon thermal transport in graphene and stanene bilayers. Phys. Chem. Chem. Phys. 19, 6554-6562 (2017).

42. Liu, B. et al. Interfacial thermal conductance of a silicene/graphene bilayer heterostructure and the effect of hydrogenation. ACS Appl. Mater. Interfaces 6, 18180-18188 (2014).

43. Han, D. et al. Phonon thermal conduction in a graphene-C3N heterobilayer using molecular dynamics simulations. Nanotechnology 30, 075403 (2019).

44. Rappé, A. K., Casewit, C. J., Colwell, K. S., Goddard, W. A. \& Skiff, W. M. UFF, a full periodic table force field for molecular mechanics and molecular dynamics simulations. J. Am. Chem. Soc. 114, 10024-10035 (1992).

45. Plimpton, S. Fast parallel algorithms for short-range molecular dynamics. J. Comput. Phys. 117, 1-19 (1995).

46. Lindsay, L. \& Broido, D. A. Optimized Tersoff and Brenner empirical potential parameters for lattice dynamics and phonon thermal transport in carbon nanotubes and graphene. Phys. Rev. B Condens. Matter Mater. Phys. 81, 205441 (2010).

47. Erhart, P. \& Albe, K. Analytical potential for atomistic simulations of silicon, carbon, and silicon carbide. Phys. Rev. B Condens. Matter Mater. Phys. 71, 035211 (2005).

48. Müller-Plathe, F. A simple nonequilibrium molecular dynamics method for calculating the thermal conductivity. J. Chem. Phys. 106, 6082-6085 (1997)

49. Hong, Y., Zhang, J. \& Zeng, X. C. Interlayer thermal conductance within a phosphorene and graphene bilayer. Nanoscale 8 , 19211-19218 (2016).

50. Liu, B. et al. Thermal transport in a graphene-MoS2 bilayer heterostructure: a molecular dynamics study. RSC Adv. 5, 29193-29200 (2015).

51. Hong, Y., Zhang, J. \& Zeng, X. C. Thermal Conductivity of Monolayer MoSe2 and MoS2. J. Phys. Chem. C 120, 26067-26075 (2016).

52. Hong, Y., Zhang, J., Huang, X. \& Zeng, X. C. Thermal conductivity of a two-dimensional phosphorene sheet: a comparative study with graphene. Nanoscale 7, 18716-18724 (2015).

53. Wu, X. et al. How to characterize thermal transport capability of 2D materials fairly? - Sheet thermal conductance and the choice of thickness. Chem. Phys. Lett. 669, 233-237 (2017).

54. Rahman, M. H. et al. Phonon thermal conductivity of the stanene/hBN van der Waals heterostructure. Phys. Chem. Chem. Phys. 23, 11028-11038 (2021).

55. Khan, A. I., Paul, R. \& Subrina, S. Thermal transport in graphene/stanene hetero-bilayer nanostructures with vacancies: an equilibrium molecular dynamics study. RSC Adv. 7, 44780-44787 (2017).

56. Noshin, M., Khan, A. I. \& Subrina, S. Thermal transport characterization of stanene/silicene heterobilayer and stanene bilayer nanostructures. Nanotechnology 29, 185706 (2018).

57. Zhang, J. et al. Phonon thermal properties of transition-metal dichalcogenides MoS2 and MoSe2 heterostructure. J. Phys. Chem. C 121, 10336-10344 (2017).

58. Taube, A., Judek, J., Łapińska, A. \& Zdrojek, M. Temperature-dependent thermal properties of supported MoS2 monolayers. ACS Appl. Mater. Interfaces 7, 5061-5065 (2015).

59. Hu, M. \& Poulikakos, D. Si/Ge superlattice nanowires with ultralow thermal conductivity. Nano Lett. 12, 5487-5494 (2012).

60. Li, D. et al. Thermal conductivity of individual silicon nanowires. Appl. Phys. Lett. 83, 2934 (2003).

61. Kong, L. T. Phonon dispersion measured directly from molecular dynamics simulations. Comput. Phys. Commun. 182, 2201-2207 (2011).

62. Xiong, S., Kosevich, Y. A., Sääskilahti, K., Ni, Y. \& Volz, S. Tunable thermal conductivity in silicon twinning superlattice nanowires. Phys. Rev. B 90, 195439 (2014).

63. Xiong, S., Selli, D., Neogi, S. \& Donadio, D. Native surface oxide turns alloyed silicon membranes into nanophononic metamaterials with ultralow thermal conductivity. Phys. Rev. B 95, 180301 (2017).

64. Li, Z. et al. Influence of thermostatting on nonequilibrium molecular dynamics simulations of heat conduction in solids. J. Chem. Phys. 151, 234105 (2019).

65. Cepellotti, A. \& Marzari, N. Boltzmann transport in nanostructures as a friction effect. Nano Lett. 17, 4675-4682 (2017).

66. Allen, P. B. Size effects in thermal conduction by phonons. Phys. Rev. B 90, 054301 (2014).

67. Liang, T., Zhang, P., Yuan, P. \& Zhai, S. In-plane thermal transport in black phosphorene/graphene layered heterostructures: A molecular dynamics study. Phys. Chem. Chem. Phys. 20, 21151-21162 (2018).

68. Li, B., Lan, J. \& Wang, L. Interface thermal resistance between dissimilar anharmonic lattices. Phys. Rev. Lett. 95, 1-4 (2005).

69. Wang, X., Zhang, J., Chen, Y. \& Chan, P. K. L. Molecular dynamics study of thermal transport in a dinaphtho[2,3-b:2',3'-f] thieno[3,2-b]thiophene (DNTT) organic semiconductor. Nanoscale 9, 2262-2271 (2017).

70. Zhang, H., Lee, G. \& Cho, K. Thermal transport in graphene and effects of vacancy defects. Phys. Rev. B Condens. Matter Mater. Phys. 84, 115460 (2011).

71. Wu, X. \& Luo, T. The importance of anharmonicity in thermal transport across solid-solid interfaces. in Journal of Applied Physics vol. 11514901 (American Institute of PhysicsAIP, 2014).

\section{Author contributions}

M. S. I. conceived the work; I. M. and A. S. M. J. I. performed the simulations under the supervision of C. S. and J. P. All authors discussed and analyzed the data and interpretation and contributed during the writing of the manuscript.

\section{Competing interests}

The authors declare no competing interests. 


\section{Additional information}

Supplementary Information The online version contains supplementary material available at https://doi.org/ 10.1038/s41598-021-04740-4.

Correspondence and requests for materials should be addressed to M.S.I.

Reprints and permissions information is available at www.nature.com/reprints.

Publisher's note Springer Nature remains neutral with regard to jurisdictional claims in published maps and institutional affiliations.

(c) (i) Open Access This article is licensed under a Creative Commons Attribution 4.0 International License, which permits use, sharing, adaptation, distribution and reproduction in any medium or format, as long as you give appropriate credit to the original author(s) and the source, provide a link to the Creative Commons licence, and indicate if changes were made. The images or other third party material in this article are included in the article's Creative Commons licence, unless indicated otherwise in a credit line to the material. If material is not included in the article's Creative Commons licence and your intended use is not permitted by statutory regulation or exceeds the permitted use, you will need to obtain permission directly from the copyright holder. To view a copy of this licence, visit http://creativecommons.org/licenses/by/4.0/.

(C) The Author(s) 2022 